\title{
LOS VALORES POLÍTICOS Y LA ORGANIZACIÓN DE LAS COMUNIDADES ESTUDIANTILES
}

\begin{abstract}
Nolberto Acosta Varela ${ }^{1}$
Resumen. Este capítulo examina la organización de los estudiantes universitarios y los valores políticos que promueven en sus centros educativos. Es importante ahondar en el estudio de los valores políticos y la organización de las comunidades estudiantiles (OCE) de los estudiantes universitarios de educación pública en Ciudad Juárez. Se toma como base, información obtenida de entrevistas semiestructuradas. También, se analizan las condiciones objetivas y subjetivas de participación estudiantil en sus centros escolares, se revisa cómo se producen o reproducen los elementos de valores políticos y organización. Se busca dar cuenta de cuáles han sido los procesos en la construcción de los instrumentos de representación a partir del marco legal, el control ejercido por las autoridades universitarias y la propia disposición de los actores en ser parte de procesos de organización y participación.
\end{abstract}

Palabras clave: Organización, Estudiantes, Valores y Política.

Abstract. This chapter examines the organization of university students and the political values being promoted in its educational centers. It is important to elaborate on the study of political values and organization of student communities (OCE) of the public university students from Ciudad Juarez. The base for this work is information taken from semistructured interviews. Also the objective and subjective conditions about student participation in their schools are analyzed, and how are political and organizational values produced or reproduced. The goal is to acknowledge which have been the processes involved in the construction of representational tools according to the legal framework, the control exercised by university authorities and the willingness from the actors to take part in the organization processes and participation.

Keywords: Organization, Students, Values and Politics.

\footnotetext{
${ }^{1}$ Doctor en Ciencias Sociales por la Universidad Autónoma de Ciudad Juárez, México. Profesor adscrito al Instituto de Ciencias Sociales y Administrativas de la UACJ.
} 


\section{Antecedentes}

Las Instituciones de Educación Superior (IES) en sus campus atienden a cientos o miles de estudiantes que constituyen comunidades diversas, tanto por su condición de género, nivel socioeconómico y compromiso hacia lo político. Al interior de dichas comunidades se "propician" condiciones para que los estudiantes universitarios desarrollen procesos políticos como los que se dan en el resto de las instituciones públicas del ámbito federal, estatal o municipal. Algunas de las situaciones relacionadas con la política tienen que ver con procesos sobre democracia, cultura política y organización de la propia comunidad estudiantil.

Las IES al igual que las instituciones del Estado, enfrenta una débil participación política que se refleja en los índices de votación cuando se trata de renovar los cuerpos colegiados de gobierno o las asociaciones de estudiantes. También se refleja una pobre Organización de las Comunidades Estudiantiles (OCE) y una escasa cultura política, todo ello, matizada por ciertos valores políticos que se recrean en los campus universitarios. En este contexto los estudiantes plantean diversas rutas de tránsito que se ubican entre lo político y su negación, lo no político o la indiferencia.

La baja participación entre los jóvenes y especialmente entre los universitarios es una situación que llama la atención, no solamente de los actores involucrados en promover una mayor participación electoral, también es una preocupación de investigadores de ciencias sociales. Por ello, ante el preocupante alejamiento de los universitarios del quehacer político en su espacio de estudio, se hace hincapié en la necesidad de conocer la situación de organización colectiva y la autonomía en la organización de la comunidad estudiantil, así como su importancia en el ejercicio de derechos y deberes universitarios encaminados a incrementar los niveles de participación política. 
Las comunidades escolares son actores políticos que a lo largo de la historia han luchado por mantener su autonomía, objetivo que no han conseguido del todo por varias razones, entre las que figuran: el diseño curricular, el modelo organizativo y político dirigido por las autoridades encargadas de la conducción institucional. La formación ciudadana desde una configuración completa es poco factible, ya que la lógica que impera en la formación de valores con respecto a los estudiantes gira en torno a las demandas del mercado y a los intereses pragmáticos de las instituciones educativas. La participación política de las y los estudiantes universitarios es considerada como limitada y muy fragmentada. Sin embargo existe y se manifiesta de diferentes maneras.

La mayoría de las investigaciones y trabajos académicos sobre los estudiantes universitarios se han realizado a partir de conflictos que se han presentado en diferente momento histórico García Cantú (1976), Guevara (1986), Sánchez (1999), Poniatowska (2001), Rosas (2001), Rivas (2007), Wences Reza (1971), Ordorika (2006), Xermeño (2008), Moctezuma (2010), Azcué (2010), Montemayor (2010), Monsiváis (2012), Muñoz (2012), Xelhuantzi (2009). No obstante, no existen suficientes estudios en México sobre la actividad política de los estudiantes universitarios al interior de sus instituciones (Durand, 1997, 2004), (Miranda 2006), otros lo han hecho a partir de un análisis de los estudiantes pertenecientes diferentes IES sobre aspectos de la cultura política nacional (Emmerich, 1998; Fernández Poncela, 1997 y 2003).

El papel que juega la educación de tercer nivel -universitaria- en los países, es determinante para su desarrollo. Podríamos decir la universidad es tan importante para el óptimo funcionamiento del mundo, como lo es la "espina dorsal” para el cuerpo humano. La educación es la columna vertebral de una sociedad, históricamente, guardando las proporciones y los niveles de desarrollo en que ésta se encontraba y las formas en que se transmitía de una generación a otra, fue determinante para permanecer, reproducirse y 
transformarse. En la actualidad, cuando se vira hacia un país o un grupo social determinado, uno de los primeros indicadores que se observan, es el de la educación.

El grado promedio de escolaridad en México es de 8.65 en población mayor de 15 años. Aún, persiste una diferencia en la relación según sexo, los hombres alcanzan 8.8 grados, mientras que las mujeres llegan a 8.5. Lo anterior, significa que no se ha superado la formación básica que es de 9 años de escolaridad. La matrícula de educación superior en México 2014- 2015 según datos de la Asociación Nacional de Universidades e Instituciones de Educación Superior (ANUIES) ${ }^{2}$ alcanzó la cifra de 2, 474, 648 estudiantes en el sector público y en modalidad de presencial, mientras que en el sector privado, llegó a 2,474, 648 de acuerdo a información de la Secretaria de Educación Pública (SEP) y la Asociación Nacional de Universidades e Instituciones de Educación Superior (ANUIES) en su anuario estadístico reporta una matrícula de 3, 718,995 estudiantes inscritos a nivel licenciatura. El Estado de Chihuahua reporta 119,171 estudiantes, Universidad Autónoma de Chihuahua (UACH) 27, 506. Universidad Autónoma de Ciudad Juárez (UACJ) 26,312.

\section{Los valores}

Cuando hacemos referencia a los valores, invariablemente, nos remite a algo que se plantea en positivo, a lo bueno, lo honesto, lo agradable, lo que tiene que ver con lo tolerante, lo justo, lo responsable y todo aquello que plasme la cara positiva de las situaciones que atravesamos como sociedad, sea en el terreno individual o colectivo. Para reproducir la mejor parte del ser humano se han construido sendas disciplinas con la intención de guiar el comportamiento individual y colectivo en las diversas áreas de desarrollo humano, la ética y la moral representan las rutas idóneas de tránsito.

\footnotetext{
${ }^{2}$ ANUIES. www.anuies.mx consultado 21 diciembre de 2015.
} 
La ética, no se refiere entonces a la realidad, sino a la conciencia individual, y lo que importa es la intención (por eso Max Weber la ha llamado Gesinnungsethik) y no el resultado. Y la moral ha de ser realizada en la sociedad y por la sociedad. La moral es constitutivamente social. La ética es, en cuanto tal, personal y social. Lo personal y lo social son primarios en ella, e inseparables en ella (L. Aranguren; 1986, pp. 16 y 19). La ética apela al deber ser, se plantea en un territorio de lo ideal, y se desarrolla bajo esa realidad, se para frente al espejo divino y quiere reflejar la perfección. En esta lógica, L. Aranguren (1986) en relación a la ética política, dice que ésta plantea que "la realidad política, debe ajustarse a un arquetipo de Estado o estado Ideal (p. 23)”.

\section{Los valores y la educación}

Dice Ricoeur (2006) que la noción de valor está cada vez más presente en nuestros discursos. Lo cual es cierto, y sobre todo en una sociedad que presenta simultáneamente varias crisis, tanto en lo económico, político, social y cultural. En este marco, México no es la excepción, los diferentes problemas que se han hecho presentes evidencian situaciones de crisis en las estructuras económicas, creando con ello visibles asimetrías entre los diferentes sectores de población, propiciando mayor desigualdad entre productores y, transformando los hábitos de consumo.

En materia política, las cosas no han marchado mejor, se ha centrado la actividad política en una transición democrática que se atoró, desde hace dos décadas se ha centrado la política en el monopolio del poder ejercido por los partidos políticos, dejando a la sociedad, solamente

el ejercicio del voto. A eso, se ha limitado el desarrollo ciudadano. Lo social, atraviesa por un problema de seguridad, que incluso en algunas regiones, -- parte de la frontera norte-- se vive una situación que la pone al borde de la anomia, debido al grado de descomposición de las instituciones responsables de mantener los parámetros mínimos de desarrollo humano y, la cultura se encuentra permeada por un conjunto de transformaciones, dependiente cada vez 
en mayor medida de estrategias mundiales que arrastran hacia la globalización que en afán de abaratar la mano de obra ha propiciado situaciones en las que la clase trabajadora ha buscado diversas maneras de complementar el ingreso, aún, con actividades ilegales.

Los valores representan una guía para moldear actitudes y comportamientos de los sujetos, sean individuales o colectivos. Casi siempre, son representados en una dicotomía (buenomalo, tolerante-intolerante, autónomo-heterónomo), están cargados de principios económicos, políticos, religiosos, culturales y sociales. En términos bourdianos, son reflejo del habitus (Bourdieu, 1989), pero como lo planteaba el propio autor, al ser procesos aprendidos en la práctica cotidiana que se desarrolla en el entorno inmediato, son diversos, dependiendo de la posición social en que se reproduzcan. Sin embargo, las actitudes, las creencias, los valores y los conocimientos forman parte de un conjunto de relaciones que se gestan en las instituciones y grupos de poder externos.

Según Alduncin (1989) la familia ocupa el primer lugar en la formación de valores, pero el segundo lugar, dice, le corresponde a la escuela. Y en ese sentido, Yurén (1995) ve a la escuela como una de las instituciones más características de la modernidad, en tanto que, procura reproducir los fundamentos objetivos y subjetivos que permitan a cada nivel, según su ubicación socioeconómica contribuir con su rol en la división del trabajo. En esa lógica, a los docentes se les ha visto como responsables de transmitir los valores que faciliten el desarrollo moral y ético de la sociedad a través del aula. Axiología y deontología "sirven” de marco para encauzar el ser y deber que se promueve por los educadores sobre los estudiantes (Haydon, 2003).

Morin (2006), habla de los imperativos contradictorios que se dan a partir de los valores, en unos casos dice, los resultados son totalmente opuestos a lo esperado, y además, dice que es imperativo propiciar una mayor complejidad que contribuya a evitar el aislamiento de los 
valores, ello se logra según él, a partir de conseguir una mayor relación entre ciencia, ética y política.

Para Ling $(2001,57)$ "los valores en la educación están determinados por la cultura a la que pertenecen”, agrega, “ningún aspecto del currículo se enseña, por así decirlo, en un vacío cultural". Es por ello que varios de los procesos nacionales o regionales que regulan los comportamientos y pausas guías tienden a reproducirse en las instituciones educativas, claro, con sus respectivas variantes, con singularidades que son producto de las autoridades y grupos de poder dominante en determinado momento histórico.

\section{Los valores políticos que promueven los directivos de la UACJ}

Roldán (2006) plantea los escenarios de formación política en las instituciones educativas y evidencia una representación silenciosa o de pocas palabras. En las sesiones de Consejo Técnico y Consejo Universitario de la UACJ, las representaciones estudiantiles terminan siempre callando, dejan de asistir a las sesiones y cuando lo hacen, son mudos presenciales, ello se debe esencialmente a que en las primeras dos reuniones se implementa una aplanadora, todo se vota por unanimidad y no se deja espacio para la deliberación, da la impresión de que todo está decidido y que allí se acude únicamente a legitimar lo acordado previamente.

\section{Libertad:}

Uno de los valores que mayor impacto pueden tener en lo político, es precisamente la libertad. La libertad de elegir, de tener autonomía e independencia. Elegir, es quizá uno de los planteamientos más amplios a los que se enfrenta la persona, ya que ello implica, la posibilidad de contar con diferentes opciones, tanto, en lo individual, así, como en lo colectivo. 
La libertad, implica la capacidad de organizarse con plena independencia de las autoridades políticas y administrativas de la institución, hacer uso de la autonomía para ensanchar las prácticas de libertad de expresión, reunión, asociación y búsqueda del poder político universitario. No obstante, la institución por su parte no solamente no fomenta que las comunidades estudiantiles actúen en ese sentido, sino, por el contrario, lo inhibe. A finales de 2015 en el campus de Ciudad Universitaria (CU) de la UACJ, se comenzaron a organizar estudiantes para protestar por un conjunto de situaciones que consideran "son injustas y les afecta" en su desarrollo universitario, la respuesta por parte de las autoridades fue de “alarma". Lo que obligó a que éstas, implementaran una serie de acciones para revertir la participación activa.

\section{Igualdad:}

La igualdad para participar políticamente como lo establece la Constitución en su Artículo 34 "todos los ciudadanos tienen derecho a ser elegidos para un cargo de elección popular", situación que no se cumple en la mayoría de las IES, incluida la UACJ, pues tiene restricciones en su Ley Orgánica y en los reglamentos del Consejo Universitario y Técnico, que ponen candados de créditos y calificaciones, violentando el marco constitucional. Al restringir la posibilidad de que cualquier estudiante acceda a la representación se rompe el principio de equidad y justicia.

Otro aspecto que violenta la igualdad de los estudiantes, es la oportunidad que se tiene para una beca trabajo, no se toman en consideración indicadores económicos o compromisos de responsabilidad familiar, por mencionar solo algunos. El criterio principal de aceptación para una beca trabajo está en relación al promedio académico del/la estudiante y la cantidad de créditos que lleva cursados, puesto que existe un candado al respecto. 
La igualdad implica reciprocidad, la cual conlleva que se dé cierto grado de relación y compromiso entre sus integrantes. Hay evidencia de que al promover las autoridades a estudiantes que deben legitimar el funcionamiento institucional, se busca sacar ventaja, implicando que la reciprocidad fomentada termine siendo negativa.

\section{Participación}

La UACJ promueve la participación estudiantil en diferentes áreas; tiene una amplia estructura administrativa para atender los requerimientos y necesidades que considera necesarios para el desarrollo estudiantil. Dicha propuesta es resultada de decisiones externas que le permitan acreditar los programas o por iniciativa propia. Es decir, la promoción de dicha participación tiene un principio, un medio y un fin, trabajando en perfecta sintonía y con una dirección perfectamente definida.

Lo que definitivamente no está en juego, es la libre participación, el debate público y la evaluación de la estructura administrativa. Cada año que se necesita renovar los consejos, es decir, los máximos órganos de gobierno, el estudiantado puede ir y votar, aunque sea con planilla única y la opción de las máquinas electrónicas del Instituto Estatal Electoral no permite la opción de otros o de abstención.

Para que la participación estudiantil sea aceptada, reconocida y apoyada, debe ser vertical, instrumentada por la propia autoridad universitaria. La otra participación, la horizontal, la que proviene de la iniciativa del estudiantado, esa, solamente será apoyada en la medida que adopte o se sume a la estructura reconocida y legal, misma que descansa en los órganos de poder universitarios. 


\section{Fraternidad:}

La universidad promueve la vinculación de los estudiantes con organizaciones de la sociedad civil, con dependencias de los tres niveles de gobierno e incluso con empresas. Esta promoción tiene como objetivo que el estudiantado realice su servicio social, que cumpla con lo estipulado en los requisitos de los programas educativos institucionales, pero, no realiza un esfuerzo extraordinario en promover programas de ayuda mutua entre los estudiantes.

No existe un programa que promueva redes de confianza entre las propias comunidades estudiantiles, ni por instituto, ni inter institutos o extensiones educativas. Cada instituto funciona como si se tratara de una universidad independiente

La institución cuenta con un programa de género y con becas para estudiantes que pertenecen a etnias que habitan en la ciudad, pero más allá de eso, no existe un programa intercultural o multicultural que entre otras cosas promueva la tolerancia.

En las universidades estadounidenses y algunas europeas es normal la existencia de fraternidades de estudiantes que sirven de apoyo y promoción de grupos. Este tipo de organizaciones tienen una vida que trasciende el tiempo que el estudiante dura en la universidad, impulsan cuestiones de raza, de género e incluso de índole económica. Son prácticas que fomentan la iniciativa, la solidaridad, la autonomía y en cierta manera ayudan a construir ciudadanía. Pero, en los campus de la UACJ, una organización de ese tipo, sería vista como subversiva. 


\section{Organización de la Comunidad Estudiantil $(\mathrm{OCE})^{3}$}

Con el concepto de Organización de la Comunidad Estudiantil, ${ }^{4}$ se hace referencia a las formas de organización en las que participan los estudiantes universitarios de la Universidad Autónoma de Ciudad Juárez (UACJ). Se refiere a la organización voluntaria de estudiantes que comparten un interés común y que se organizan a partir del mismo. Es una alianza entre singularidades que trasciende esa condición en aras de influir políticamente en la esfera universitaria. La OCE, pretende influir con el proyecto que ganó, participar activamente en el desarrollo de actividades de índole académico, cultural, deportivo y político.

La Organización de la Comunidad Estudiantil es considerada como el espacio propio en el que participan los estudiantes para representar sus intereses, en este caso se usa el concepto de OCE como símil de la sociedad civil, para referirse al entorno de universidad. La organización estudiantil, asume diversas formas, unas, como interlocutores ante conglomerados de estudiantes que organizan por facultades, institutos, programas e incluso, como representantes de toda la universidad. Los nombres van desde, consejos estudiantiles, sociedades de alumnos, comités estudiantiles, hasta, federaciones de estudiantes.

La organización estudiantil, es el instrumento que permite a los estudiantes se posicionen, son representaciones formales e informales: a) de los órganos formales de gobierno, asociaciones estudiantiles, consejos universitarios, colegios, clubs; b) informales, asociaciones estudiantiles independientes; células o redes que buscan ciertos objetivos dentro de los centros educativos. La posición política de la organización estudiantil puede estar orientada por principios ideológicos, investigación u cualquier otro interés; puede tener la

\footnotetext{
${ }^{3}$ El concepto que uso para dar cuenta de la Organización de la Comunidad Estudiantil surge de la teoría y la práctica de la sociedad civil, por lo que reconozco la deuda con los planteamientos que hicieran en su momento Hegel, Gramcsi, Bobbio, entre otros. De los autores más recientes me apoyo en Cohen y Arato (2001), Olvera (2003), Fernández (2003) y muchos más. Se planteó que "la sociedad civil y el Estado fungen como dos momentos necesarios, separados pero contiguos, diferentes pero interdependientes del sistema social en su complejidad y en toda su articulación" (Bobbio, 1992, p. 62).

${ }^{4}$ La Organización de la Comunidad Estudiantil se da mediante asociaciones estudiantiles, sociedades de alumnos u cualquier tipo de colectivo diferente a la estructura de gobierno universitario.
} 
intención de influir dentro o fuera de la institución educativa. Empero, la organización estudiantil es por conveniencia un excelente recurso para plantear sus demandas, para tomar partido, para legitimar o no las decisiones sobre política educativa de la institución. La organización de la comunidad estudiantil puede propiciar cierto grado de conciencia, suscitar como parte de su trabajo cotidiano la participación activa.

La Organización de la Comunidad Estudiantil, además de agrupar a los estudiantes en estructuras que les permitan conformar la representación, ayuda a la discusión y solución de sus necesidades académicas, culturales, sociales, deportivas y políticas entre otras. La esencia de toda OCE, conlleva una perspectiva política, ya que uno de sus principios, implica, concentrar intereses individuales y transformarlos mediante el cuerpo colegiado en intereses colectivos de los estudiantes. Es un espacio de participación que debe constituirse mediante procesos democráticos, autónomos y plurales.

De esta manera, la OCE se debe plantear como un espacio independiente y autónomo que se proponga el fortalecimiento de las identidades estudiantiles. Las OCE son asociaciones que buscan impulsar condiciones incluyentes de participación dentro de la comunidad universitaria. Es una forma de organización de la comunidad universitaria que busca identidad y ha decidido voluntariamente procesar sus necesidades escolares bajo principios democráticos de participación.

Todas las Instituciones de Educación Superior (IES) contemplan una estructura administrativa para atender y canalizar las necesidades de los estudiantes. Lo cual no implica que los estudiantes constituyan sus propias organizaciones; las intenciones e intereses del gobierno universitario no siempre cubren o satisfacen la pluralidad de los intereses estudiantiles.

En la política contemporánea se da la formación de organizaciones al margen del estado y de los partidos políticos, las llamadas Organizaciones de la Sociedad Civil (OSCs), o Tercer Sector, y las Organizaciones No Gubernamentales (ONGs), que son algunas de las formas en las que la sociedad decide participar políticamente como apoyo o contrapeso de las 
instituciones del estado. En las IES, las OCE son en muchos casos, mecanismos de organizaciones constituidas como alternativa hacia el poder político universitario ${ }^{5}$, puesto que buscan conducir los derechos políticos, participar activamente en la vida políticauniversitaria y organizarse de diferentes formas, con múltiples finalidades.

Las OCE, son la condición de la vida asociativa que representa los intereses de los estudiantes universitarios, es la forma en que los estudiantes pueden dialogar colectivamente, incrementar su poder con respecto al resto del poder político universitario y promover una que favorezca su colectividad. Es comprensible que los estudiantes universitarios defiendan sus propios intereses y actúen de diversas formas, en agrupaciones o asociaciones dentro y fuera del ámbito escolar. Por otra parte, se estima que hay un buen porcentaje de universitarios que permanecen al margen de participación colectiva.

El énfasis en la organización política de los estudiantes universitarios, como forma de posicionamiento del alumnado ante las estructuras formales de poder institucional, permite pensar a éstas como realidades autónomas y de participación, pero no siempre funcionan así, pueden ser instrumentos de control de las mismas autoridades para el status quo. De antemano, se entendería que las OCE debieran tener plena autonomía política que les permitiera autoconstituirse y autoregularse. La autoridad universitaria tendría que ser muy respetuosa en garantizar la organización estudiantil, su diversidad y funcionamiento, así como fomentar el diálogo entre los estudiantes organizados y los no organizados. También, el gobierno universitario debe aspirar a construir escenarios de deliberación y coordinación con las OCE.

No sobra enfatizar e insistir en que las OCE deben tener la capacidad de tomar decisiones en función de procesos deliberativos y democráticos; deben aceptarse como organizaciones

\footnotetext{
5 Sobre el "poder político universitario" se toma como referente la consideración que desarrolla Acosta (2006, p. 150) de "régimen político" para referirse a la estructura de acceso y distribución del poder en la Universidad. Ya que dice que permite "establecer la relación entre valores, reglas, mecanismos y figuras de autoridad que estructuran todo régimen político"
} 
que actúan, no al margen del poder político universitario, pero sí, con la autonomía suficiente. En el entendido, que no son parte de la estructura formal del poder político universitario, ya que no son autoridad, pero sí, organización política constituida frente al poder de gobierno universitario. En coincidencia con Olvera (2003), haciendo el símil de las OCE, con las OSC o tercer sector, estas son formas de organización que permiten la defensa de los derechos, en este caso de los estudiantiles universitarios, además de ampliar el horizonte de la política estudiantil.

\section{LAS OCE EN LA UNIVERSIDAD AUTÓNOMA DE CIUDAD JUÁREZ}

La UACJ, es una institución de aproximadamente 28,000 estudiantes repartidos en cuatro institutos, seis campus, de los cuales dos están en otras ciudades, uno en Nuevo Casas Grandes y otro en Cuauhtémoc, ambas dentro del estado de Chihuahua. En la UACJ, los estudiantes se organizan mediante sociedades de alumnos con adscripción a un programa determinado, no están reglamentadas, es decir, no existen en la Ley Orgánica o en alguno de sus reglamentos internos. Su constitución y funcionamiento, obedece más a la necesidad del programa para sus evaluaciones y al interés de los propios estudiantes para canalizar actividades de colegio.

De las 67 licenciaturas que se ofertan en la totalidad de campus universitarios se cuenta con los dedos de las manos los programas que tienen una organización estudiantil y sobran dedos. No existe una cultura de participación organizada entre el alumnado, tampoco, se promueve desde las autoridades universitarias. Éstas se encargan de promover la participación estudiantil mediante sus mecanismos oficiales, de los cuales el más importante es:

La Dirección de Extensión y Servicios Estudiantiles se creó con el fin de acompañar al estudiante de todos los grados en cada etapa de su desarrollo como miembro de la comunidad estudiantil, brindándole apoyo, información, asesoría y gestión, con el objeto de coadyuvar en la formación de Profesionistas Líderes con Calidad humana. Queremos que estudiar en la Universidad Autónoma de Ciudad Juárez sea una experiencia de formación Integral que vaya a la vanguardia de México y del mundo (UACJ, 2015. http://www.uacj.mx/). 
Mediante esta dirección general de atención a los estudiantes, se promueven un conjunto de actividades de manera institucional, mismas que son canalizadas a través de áreas creadas especialmente para su atención, una de ellas es la subdirección de bienestar estudiantil que se encarga de:

1. Brindar servicios de apoyo social al estudiante, mismos que van a proporcionar los medios necesarios para el éxito académico del estudiante.

2. Promoción de la salud integral, desde una perspectiva muy amplia en la que se dé una interacción entre la salud física, mental y las relaciones con los demás, para mantener un equilibrio entre esta tríada, y así alcanzar niveles óptimos de rendimiento escolar.

3. Promover la recreación y el sano esparcimiento para el aprovechamiento productivo del tiempo disponible del estudiante, y así disminuir la tensión, producto de la presión escolar.

4. Fortalecer el desarrollo humano y crecimiento personal del estudiante a través del desarrollo de habilidades, destrezas y capacidades para el buen desempeño social y profesional $^{6}$.

También, se cuenta con una subdirección deportiva que canaliza y promueve las actividades relacionadas con los deportes que practican las y los estudiantes en sus diferentes modalidades y categorías, sea, como simple disciplina de entretenimiento, amateur o profesional. A través de una dirección y una coordinación generales se canalizan las gestiones que tienen que ver con el quehacer estudiantil: servicio social, movilidad, prevención y atención de salud inicial, artes, oficios y un conjunto de actividades.

\footnotetext{
6 Universidad Autonoma de Ciudad Juárez, Servicios escolares
} http://www.uacj.mx/DGESE/SE/Paginas/default.aspx\#tab-1. consulta, 12 de enero de 2016. 
Dice Olvera (2003, p. 25) que "el fortalecimiento de la sociedad civil depende del desarrollo de las asociaciones voluntarias que forman su tejido social y materializan nuevas formas de solidaridad". En una analogía de la sociedad civil, con lo que representan las OCE en la UACJ, queda claro que: es necesaria, la construcción de todo tipo de organizaciones estudiantiles que representen los diferentes intereses y que éstas se organicen en torno a sus propias necesidades.

Convengo con el planteamiento de Olvera, que considera a la sociedad civil como "un proceso en continua renovación y en constante lucha contra el autoritarismo, la ilegalidad y la antidemocracia" (Olvera, 2003, p. 39). Las OCE deben tener, además de esos compromisos, el de la autonomía plena; situación difícil de darse con organizaciones actuando dentro del marco institucional.

Dentro de la UACJ, fue constituido el Comité Universitario de Izquierda (CUI, véase Cuadro 1), organización independiente influenciada con una ideología de izquierda trotskista. Es una organización politizada que tuvo una presencia visible durante los años de la violencia exacerbada en la ciudad, participaba con organizaciones en lucha por la defensa, justicia y aparición con vida de las mujeres desaparecidas, también, emprendieron una lucha contra la militarización ${ }^{7}$. En este marco, realizaron con frecuencia foros y eventos sobre las problemáticas más sensibles en la ciudad.

\footnotetext{
${ }^{7}$ Asamblea ciudadana juarense. http://www.noalamilitarizacion.blogspot.mx/. consulta 26 enero 2016.
} 


\section{Cuadro 1. Comité Universitario de Izquierda (CUI).}

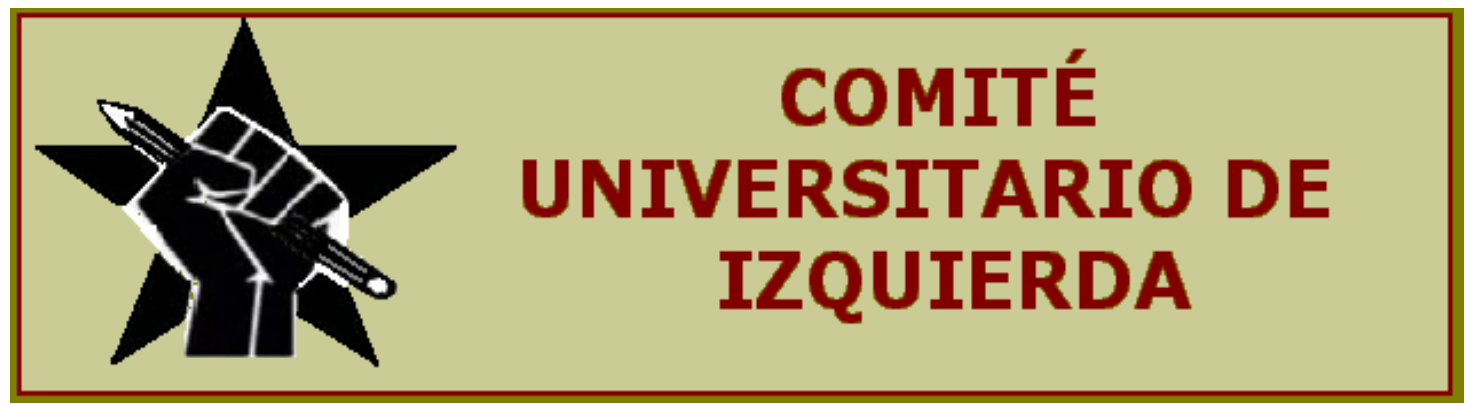

Fuente: Comité Universitario de Izquierda. Disponible en http://comiteuniversitariodeizquierda.blogspot.mx/

Con el surgimiento del movimiento estudiantil \#yo soy 132, lograron involucrarse y dominar la dirección local del mismo, hasta el agotamiento de éste, lo que les acarreó algunas críticas. Una de las banderas de esta organización en la comunidad universitaria se dio con respecto a las cuotas que cobra la institución educativa ${ }^{8}$.

Los integrantes del CUI, se involucraron con el movimiento surgido por la desaparición y posible asesinato de los 43 estudiantes normalistas de Ayotzinapa. En torno a este trágico acontecimiento que indignó al país, se pusieron al frente del movimiento que en la ciudad exigía la aparición de los estudiantes con vida; y, como en otros movimientos anteriores, se logró una buena participación de la comunidad universitaria, lo que llevó al movimiento a exigir al rector que se pronunciara a favor de sumarse al paro nacional, al negarse éste, fueron tomadas las instalaciones de rectoría de la UACJ.

\footnotetext{
${ }^{8}$ Asamblea por la reducción de cuotas de inscripción. http://noalasaltascuotas.blogspot.mx/. consulta 26 enero 2016.
} 


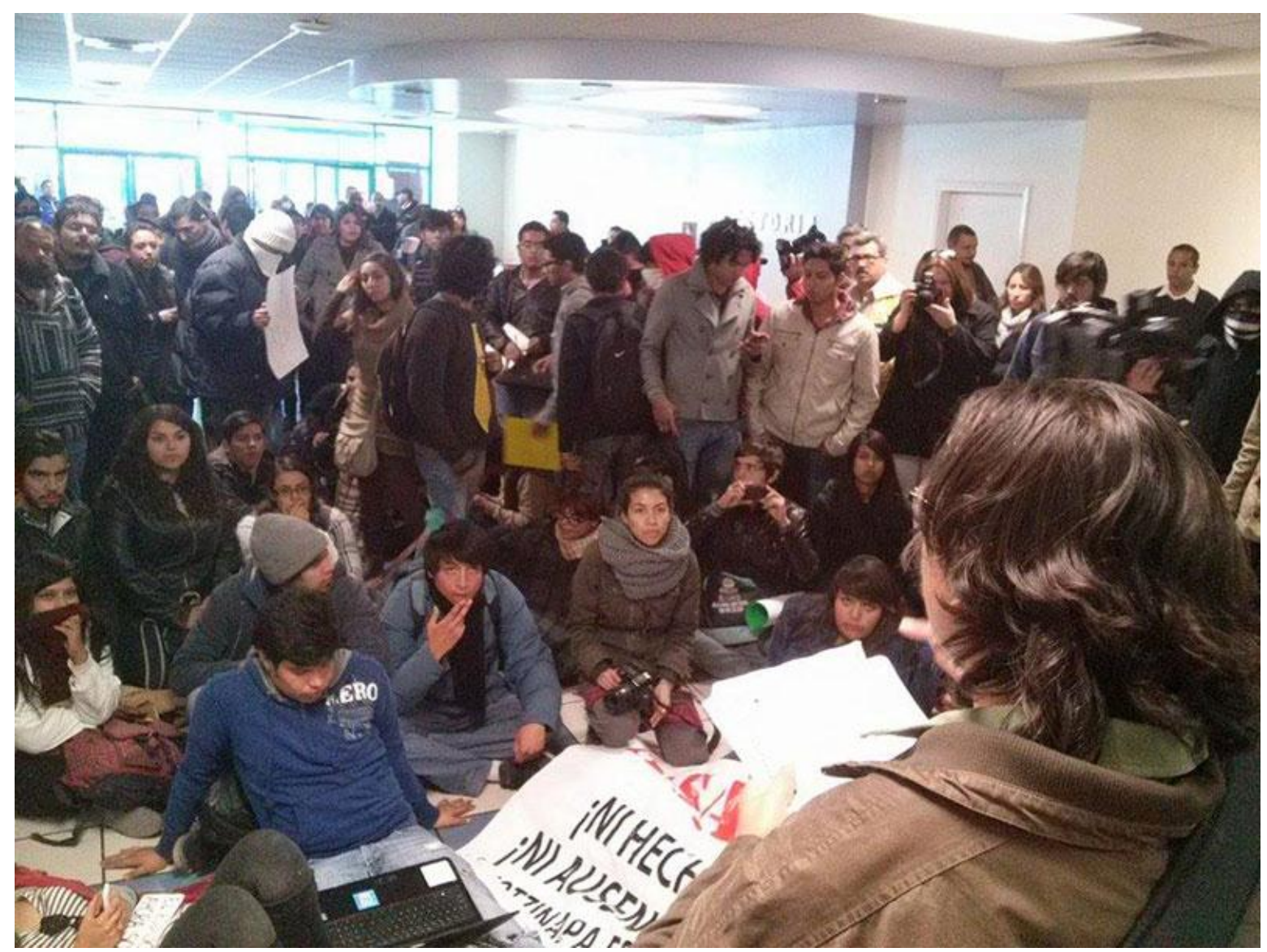

Fuente: Diario 19.

La toma de la rectoría de la UACJ se dio mediante la Asamblea Universitaria ${ }^{9}$, organización creada ex profeso, para dirigir el movimiento de Ayotzinapa en Ciudad Juárez. Desde finales de 2015 los integrantes del CUI participan activamente en las huelgas obreras de algunos “operadores de producción” de cuatro maquiladoras en la localidad ${ }^{10}$. A partir de 2016

\footnotetext{
${ }^{9}$ Sociedad Civil Organizada, Juárez Dialoga Revista semanal. Recuperada de http://juarezdialoga.org/boletininformativo/comunicado-1-asamblea-universitaria-de-la-uacj/.

10 Despide maquiladora de Juárez a 125 obreros por denunciar abusos recuperado de http://www.jornada.unam.mx/2015/08/14/estados, Paro en la maquila Foxconn en Ciudad Juárez disponible en http://www.laizquierdadiario.com/Paro-en-la-maquila-Foxconn-en-Ciudad-Juarez. Consulta 27 enero 2016.
} 
iniciaron una campaña promoviendo a trabajadoras y trabajadores de este movimiento obrero para que participen en la contienda electoral por la candidatura independiente para el ayuntamiento de Ciudad Juárez. ${ }^{11}$

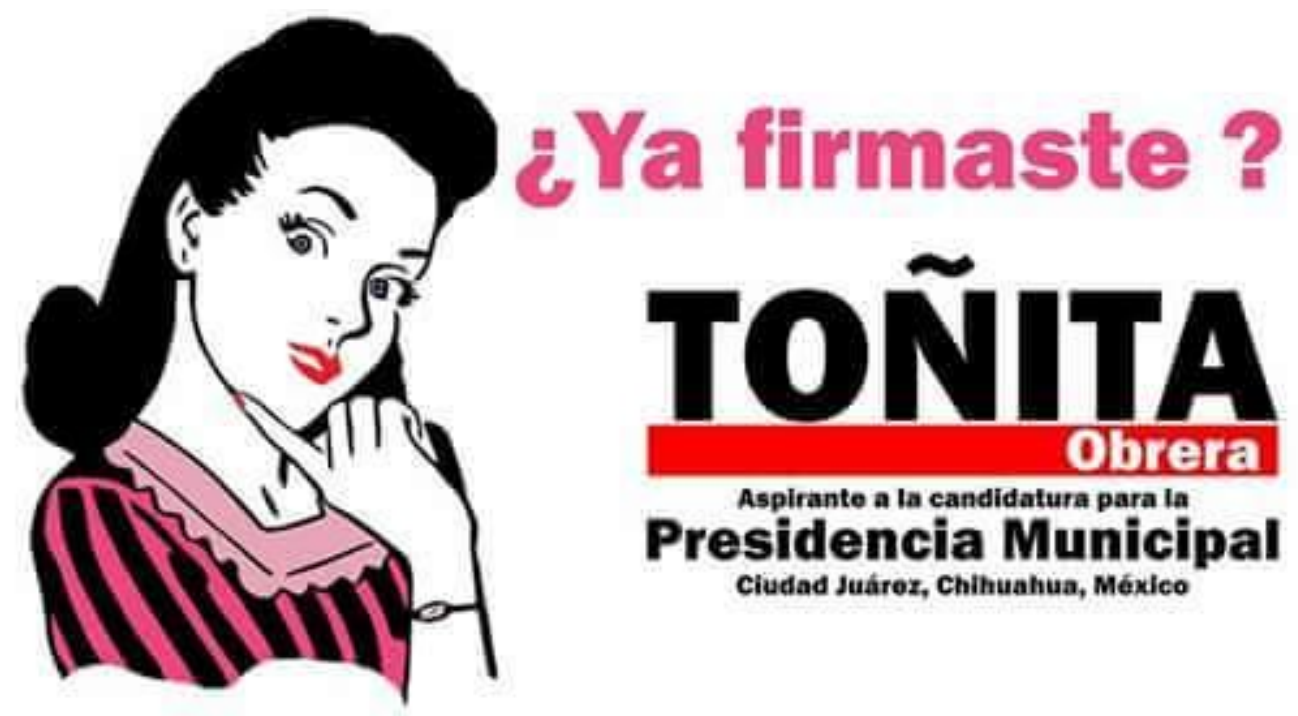

Fuente: Facebook Yo firmo por Toñita.

Ahora es "Toñita", en la elección anterior resucitaron al "Burro Chón", candidato que usaba el movimiento urbano popular denominado Comité de Defensa Popular (CDP) -hoy parte del Partido del Trabajo (PT)- en los ochenta para participar parodiando los procesos electorales.

Los integrantes del CUI, iniciaron actividades políticas desde la universidad a mediados de la década anterior, sus principales integrantes ya concluyeron una primera licenciatura y algunos de ellos realizan una más, sin embargo, aun cuando han logrado incorporar nuevos integrantes universitarios, la dirigencia no se ha renovado completamente. Pero, más allá de esta organización universitaria en la UACJ, difícilmente ha germinado alguna otra forma de

\footnotetext{
${ }^{11}$ Facebook: Yo firmo por Toñita disponible en https://www.facebook.com/Yo-Firmo-por-To\%C3\%B1ita554053818083951/?fref=photo .Consulta 2 febrero 2016.
} 
organización universitaria que funcione con regularidad y que cuente con autonomía en sus decisiones.

Su participación sobre situaciones que afectan a la sociedad en general, se encaminan con mayor frecuencia a problemáticas relacionadas con la violencia.

Cuando la organización plantea entre sus objetivos representar los intereses de las comunidades implicadas, asume un carácter político que se refleja en el posicionamiento ante las autoridades en cuestión.

Es obvio que las OCE no figuran como mediadores entre la base estudiantil y las autoridades universitarias, por una parte, la institución promueve una participación de sus bases estudiantiles, pero lo hace de la siguiente manera:

- Para nosotros es muy importante que los estudiantes se organicen en equipos deportivos, ahorita, estamos promoviendo un torneo de futbol, nos interesa que formen equipos los estudiantes de los primeros tres semestres y poder sacar adelante un buen torneo, para ello, estamos pasando de salón en salón e invitar a que se organicen (coordinador deportivo ICSA).

Para los directivos de la institución, la organización estudiantil significa la compartición del poder, la necesidad de rendir cuentas y la politización de la comunidad estudiantil, es por ello que hacen todo lo posible por mantenerlos marginados. Participación sí, pero no organizada por los propios estudiantes, sino por los órganos administrativos de la propia institución.

Cuando se trata de organizaciones estudiantiles de carácter político se restringen los espacios físicos donde las OCE puedan tener contacto permanente con el resto de la comunidad estudiantil, evitando con ello su consolidación:

- La función principal de una sociedad de alumnos, es, no tanto el abogar por el bienestar de unas cuantas personas, sino del conglomerado entero de estudiantes. Es muy difícil que los estudiantes puedan mantenerse unidos, hay muchas diferencias de intereses, mismos que se hacen patentes conforme se avanza en la carrera, llámese a esto desde el hecho de tener un conflicto o algún problema al buscar algún fin dentro de la universidad; desarrollarse en un proyecto, desarrollarse académicamente. (Estudiante ICB). 
Los estudiantes que participan en una OCE lo hacen porque quieren "trabajar en pro de la comunidad estudiantil", Les interesa apoyar el bienestar, orientar, organizar, velar... por el interés del conjunto del estudiantado:

-Nos interesa que los estudiantes promuevan distintos proyectos, buscar participar en congresos nacionales e internacionales, participar en redes y asociaciones universitarias de todo el país. Hacer gestión ante la institución o donde sea posible para beneficio de los estudiantes. (ICSA- Asociación Estudiantil).

Instituciones como la UACJ no tienen contemplado en su esquema legal una estructura para la atención de las necesidades estudiantiles que no sea la que se desprende de sus estructuras burocráticas. Tienen claro, que los recursos humanos, técnicos y materiales no pueden venir de la voluntaria asociación de la comunidad estudiantil.

La democracia que se promueve en la institución es vertical, la representación de los consejos técnicos y universitario surgen a propuesta de los diferentes niveles de gobierno universitario. En la más reciente elección para conformar dichos órganos de poder la situación no fue diferente. Solamente en el programa de Derecho participaron tres fórmulas para el Consejo Universitario; obtuvieron la mayoría de votos un estudiante y su suplente que no eran de la planilla oficial. La experiencia fue que este consejero mantuvo un alto perfil de participación en las sesiones del Consejo Universitario, a diferencia del resto de sus condiscípulos que llegaron por la vía oficial.

Para que las OCE funcionen con regularidad deben contar con la infraestructura necesaria que les permita hacer frente a los requerimientos de la comunidad estudiantil. Pero, indudablemente, lo más relevante debe ser la tolerancia, el reconocimiento de éstas por parte de la institución educativa y el libre ejercicio con autonomía. La ausencia de OCE, promueve la consolidación del autoritarismo y antidemocracia.

En general, la existencia de OCE en la UACJ depende de la buena voluntad que tengan los coordinadores de los programas para su constitución, renovación y funcionamiento. No 
existe una regularidad en las organizaciones estudiantiles, algunas de ellas sirven más para los procesos evaluativos de los programas, que como instrumentos de participación.

Los estudiantes de la UACJ se organizan de diferentes formas: en Medicina lo hacen a través de una revista, de un comité que se encarga de la participación en congresos y de un comité que participa en la revisión bibliográfica. Los estudiantes del Programa de Derecho en el Instituto de Ciencias Sociales y Administrativas (ICSA) han marcado la diferencia en cuanto a la participación en la disputa por las sociedades de alumnos y la competencia por los consejos estudiantiles institucionales. En la elección para el Consejo Universitario en noviembre de 2015, la planilla que triunfó no fue la oficial. El Consejero Universitario propietario dijo lo siguiente:

Cuando nos inscribimos, nadie pensaba que fuéramos a ganar, empezamos a platicar con nuestros compañeros, pasamos a los salones y todo mundo nos decía que la planilla de la dirección era la buena. Seguimos promoviendo nuestra propuesta y finalmente triunfamos. Lo curioso, era que por mi forma de vestir no nos daban muchas posibilidades (ICSA-Consejero de Derecho).

El joven que ganó la elección de Consejero Universitario, viste de vaquero, usa sombreo y botas. Le han comentado que hacía varios años no se presentaba competencia; tenía tiempo presentándose planilla única. Esa práctica de planilla única es una costumbre bastante arraigada en la cultura política de la Institución. En 2007 en ICSA se dio competencia en los programas de Educación, Derecho y Administración, aunque la competencia estuvo muy reñida, en los tres programas, solamente triunfó como independiente al oficialismo la fórmula al consejo Técnico por educación (Acosta 2013).

En la UACJ existe una obsesión por la unanimidad en las sesiones de Consejo, todas las resoluciones se sacan con la aprobación absoluta, no existe la diferencia, esa búsqueda de unanimidad es lo más parecido a los sistemas políticos absolutistas y totalitarios.

En el Instituto de Ingeniería y Tecnología (IIT) las sociedades de alumnos o consejos estudiantiles como formas de OCE, simplemente no forma parte de las dinámicas de 
organización horizontal y de abajo hacia arriba. Para la participación de los Consejos Técnico y Universitario, los estudiantes que forman parte de la elección de las planillas -únicas- son llamados a participar por los responsables de los programas educativos en cuestión.

En el Instituto de Arquitectura, Diseño y Artes (IADA) se dan condiciones muy semejantes a las que se presentan en IIT. Si bien es cierto que los estudiantes de IADA tienen un perfil muy diferente al de las ingenierías, el comportamiento político estudiantil no lo es tanto. Las comunidades estudiantiles se organizan para asistir a congresos, seminarios y otras actividades académicas y culturales, pero no constituyen asociaciones que sobrepasen la estancia de los propios estudiantes en su campus.

Los estudiantes de Ciudad Universitaria, Nuevo Casas Grandes y Cuauhtémoc no tienen derechos políticos, no cuentan con representantes ante los Consejos Universitario y Técnico. En la conformación de OCE, no existe evidencia de conformación de ningún tipo de organización. Se menciona con mucha insistencia en las redes sociales la percepción de ser discriminados y contar con docentes menos preparados. Sin embargo, se han presentado conatos de organización espontánea que ha evidenciado la falta de atención a sus demandas. Las prácticas de participación política-estudiantil son diferentes en cada instituto, ICSA a lo largo de la historia de la universidad ha sobresalido en el terreno de la disputa por los espacios de organización y poder, seguido de ICB, donde se ha hecho lo propio, pero no así en IADA e IIT. Se puede decir, que existe una pobre cultura de participación y de lucha por los espacios de representación estudiantil en la UACJ.

Mouffe (2003), considera como un obstáculo preocupante para la consolidación de la democracia, que los principios políticos de libertad e igualdad están muy lejos de ser prácticas reales. Por lo menos en la UACJ, se ven lejanas las comunidades estudiantiles pensadas como asociaciones constituidas libremente. Los derechos políticos de los miembros de la comunidad estudiantil quedan a resguardo de las autoridades, en dado caso que se permita el funcionamiento de una sociedad o consejo estudiantil, se acepta que la misma deba 
representar a todo el universo de estudiantes del programa, instituto, facultad o escuela para la que fue autorizada. No se toma en cuenta que son los estudiantes quienes tienen el derecho de igualdad y de libertad para constituir cuantas OCE les sean necesarias para encauzar sus intereses.

Para Fernández (2003), la democracia es una forma determinada y específica de organización política. En esta lógica, la mejor forma de impulsar la democracia dentro de la comunidad universitaria, es haciéndolo mediante instrumentos de organización estudiantil. Bobbio (1992) invocó que la democracia y la participación se ejercieran en todos los espacios y lugares donde se desenvolviera el individuo: la escuela, los sindicatos y todas aquellas esferas que implicaran la participación de muchos. Decía, que "son un lugar idóneo para el ensanchamiento democrático.

\section{Conclusión}

Los valores políticos y la Organización de la Comunidad Estudiantil en la UACJ son asignaturas que deben ser analizadas a mayor profundidad. Por una parte es importante no perder de vista las características de sus estudiantes, el perfil de los programas y la actitud de los docentes. La autoridad, ha seguido un patrón de comportamiento desde sus inicios en 1973, no debe soslayarse que fue un modelo universitario surgido bajo el esquema departamental que replicó el modelo chileno y de la Universidad Autónoma Metropolitana (UAM), como respuesta a los acontecimientos de 1968.

Aun cuando la UACJ, cuenta con una población estudiantil de aproximadamente 29000 alumnos, su vocación de gobierno es patriarcal, se maneja como si se tratase de un gobierno de familia, y de familia muy conservadora, en la cual se cuida mucho tener el control sobre sus miembros en una estructura de índole piramidal. Ello le ha permitido mantener una forma de gobernabilidad muy "eficiente", con grupos de poder muy cerrados y con un conocimiento de las reglas escritas y no escritas que le dan funcionalidad. 
En relación a los valores políticos que funcionan en la institución, son los que la universidad se encarga de promover. Respecto a los que promueven una perspectiva diferente a la línea institucional, son simplemente descartados, invisibilizados o incluso descartados. La autonomía, la independencia, la equidad la tolerancia, la participación, la reflexibilidad, la fraternidad y la igualdad, son algunos de los valores que están muy limitados y se articulan solamente cuando son impulsados por la institución y con las condiciones que ésta pone.

Un enorme déficit se evidencia en cuanto a la Organización de la Comunidad Estudiantil se refiere. Al no estar legisladas formas de organización estudiantil; al no contar con espacios para estos menesteres y no destinar un presupuesto para ello; la organización, simplemente es incipiente y sumamente reducida. La universidad tiene el monopolio en materia de organización, además, lo hace evidente y utiliza su estructura administrativa para cubrir o rellenar cualquier resquicio que surge. El estudiantado por sí solo, tiene pocas posibilidades de impulsar proyectos de organizaciones autónomas e independientes. 


\section{Referencias}

Acosta Varela, N. "La ciudadanía política de los estudiantes universitarios en Ciudad Juárez: Universidad Autónoma de Ciudad Juárez, Facultad de Ciencias Políticas y Sociales de la Universidad Autónoma de Chihuahua e Instituto Tecnológico de Ciudad Juárez, México, Universidad Autónoma de Ciudad Juárez”. (2013).

Almond, G. y S. Verba "The civic culture". Political attitudes and democracy in nations. Estados Unidos, Princenton University Press. (1963).

Apple, M.W. y Beane, J.A. "Escuelas democráticas”. España, Editorial Morata. (Comp.)(1997).

Arendt, Hannaha. "La condición humana”. Paidós, España, 2005.

Azcué Bilbao, K. "El movimiento estudiantil poblano (1952-1957)”, entrevista con Francisco Arellano Ocampo. México. Benemérita Universidad Autónoma de Puebla. (2008).

Bobbio, N. "Liberalismo y democracia”, Breviarios Fondo de Cultura. (1991).

Económica, México, 1991.

(1992). “El futuro de la democracia”. México. Fondo de Cultura Económica.

Bolívar, A. Educación para la ciudadanía. España, Editorial Graó, (2007).

Bourdieu, P. Prólogo: Estructuras sociales y estructuras mentales en: Bourdieu, Pierre. "La nobleza de Estado". Grandes Ecoles y espíritu de cuerpo, Paris: Minuit, (1989),

Cohen, J. L, y A. Arato. "Sociedad civil y teoría política". México: Fondo de Cultura Económica. (2001).

Constitución Política de los Estados Unidos Mexicanos. "México, Trillas". (2010).

Durand Ponte, V. Cultura política de masas y el cambio del sistema político: El papel de la ambigüedad cultural. Revista Mexicana de Sociología, enero-marzo de 1997, año LXI, núm. 1, pp. (1997).

."Ciudadanía y cultura política en México 1993-2003”. México, Siglo XXI Editores, (2004).

Del val, J. “Hacia una escuela ciudadana”. España, Morata. (2006).

De Mora, J. C.E.U. vs U.N.A.M. ¿La hora del neofascismo? México. EDAMEX (Editores Asociados Mexicanos, S.A.) (1987).

ENCUP. Secretaría de Gobernación. (2010).

Encuesta Nacional de la Juventud. Instituto Mexicano de la Juventud (2010).

Espejel, J. P. “La crisis universitaria de 1935”. En, Guevara, G. “Las luchas estudiantiles en México”. México, Editorial Línea, (1986).

Fernández Poncela, A. “Hombres, mujeres y política”. Una mirada desde la opinión pública y sus protagonistas. México: UAM-Xochimilco. (1997).

. "Cultura política y jóvenes en el umbral del nuevo milenio". México: IFE, SEP e Instituto Mexicano de la Juventud, (2003).

Fernández Santillán, J. “El despertar de la sociedad civil”. México, Océano. (2003).

García Cantú, G. Javier Barros Sierra 1968. México, XXI Siglo Veintiuno Editores S.A. (1976).

González, Rubio I. J. (Coordinador). “México, 30 años en movimiento”. México; Universidad Iberoamericana, (1998).

González, Villarreal, R. "El acontecimiento \#yo soy 132, crónicas de multitud”. México, Editorial Terracota ET. (2013) 
Guevara, Niebla, G. “Las luchas estudiantiles en México”. México, Editorial Línea Libro de Gilberto Guevara Niebla, (1986).

."La democracia en la calle: crónica del movimiento estudiantil mexicano". México, Siglo XXI. (1988)

Gimeno, Sacristán, J. “Educar y convivir en la cultura global”. España, Morata. (2002).

Haydon, G. "Ensañar Valores, Un nuevo enfoque”. España, Morata, Centro de Investigación y Documentación Educativa y Ministerio de Educación Cultura y Deporte. (2003).

Heslep, R. D. “La educación en la democracia, la función moral de la educación en el estado democrático”. Argentina, Grupo Editorial Latinoamericano. (1993).

Monsivaís, C. "El 68 la tradición de la resistencia”. México, Ediciones ERA. (2012).

Moreno, Yaim W., Vázquez Duarte, M., Xelhuntzi, Tesiu R. y otros. "Yo soy huelguista y soy de la UNAM". Análisis y reflexiones sobre el movimiento universitario de 19992000. México; Editorial Redez, “Tejiendo la Utopía”. (2009).

Mouffe, Ch. "El retorno de lo político: comunidad, ciudadanía, pluralismo, democracia radical". Argentina, Paidós. (1999).

Mouffe, Ch. "La paradoja democrática". España, Gedisa Editorial Punto Crítico. (2003).

Muñoz, Tamayo, V. "Generaciones". Juventudes universitarias e izquierdas políticas en Chile y México (Universidad de Chile-UNAM 1984-2006). Chile, LOM Ediciones. (2012).

Olvera, A., J. “Sociedad civil, esfera pública y democratización en América Latina”. México: Universidad Veracruzana y Fondo de Cultura Económica. (2003).

Ordorika, I. "La disputa por el campus". Poder, política y autonomía en la UNAM. México, Universidad Nacional Autónoma de México y Plaza y Valdés. (2006).

Payá, Sánchez, M. “Educación en valores para una sociedad abierta y plural: aproximación conceptual”. España, Desclée De Brouwer. (1997).

Poniatowska, E. “La noche de Tlatelolco”. México, Biblioteca Era. (2001).

Quintana, Cabañas, J. "Pedagogía axiológica, la educación ante los valores”. España, Dykinson. (1998).

Roldán, Vargas, G. "La institución educativa: escenario de formación política de niños, niñas y jóvenes". En, Tonon, G. (compiladora). "Juventud y protagonismo ciudadano". Argentina. Espacio. (2006).

Rosas, M. "Plebeyas batallas". La huelga en la Universidad. México, Ediciones Era. (2001).

Rivas, Ontiveros, J. “La izquierda estudiantil en la UNAM”. Organizaciones, movilizaciones y liderazgos (1958-1972). México, Universidad Nacional Autónoma de México y Miguel Ángel Porrúa. (2007).

Santos, Guerra, M. (2002). Hacia el Futuro: riesgos y esperanzas, en López Rodríguez, Francese. Valores escolares y educación para la ciudadanía.España, Editorial Laboratorio Educativo.

Savater, F. “La educación y los valores de la ciudadanía”. En, Órnelas, Carlos (compilador). "Valores, calidad y educación". México, Editorial Santillana. (2002).

Scherer, García, J. y C. Monsiváis. "Parte de Guerra Tlatelolco 1968”. México, Nuevo siglo, Aguilar. (1999).

Valderrama H., C. “Ciudadanía y comunicación”. Colombia:Universidad Central, IESCOUC y Siglo del Hombre. (2007).

Yurén, Camarena, M. Etnicidad, valores sociales y educación. Universidad Pedagógica Nacional, México, 1995. 
Yus, Ramos, R. “Temas transversales y educación en valores: la educación del siglo XXI”. En, López Rodríguez, Francesc (Director de colección). "Valores Escolares y educación para la ciudadanía". España, Editorial Laboratorio Educativo. (2002).

Wences, Reza, R. "El Movimiento estudiantil y los problemas nacionales”. México, Editorial Nuestro Tiempo. (1971). 